Background In Peru, syphilis disproportionally affects men who have sex with men (MSM) and male-to-female transgender women (TW) with prevalence rates as high as $21 \%$. To our knowledge, there are no available data describing circulating strains of T. pallidum in Lima, Peru. We used the CDC subtyping scheme to identify T. pallidum circulating subtypes among MSM and TW from two STI clinics in Lima, Peru.

Methods A cohort of 401 MSM and TW were assessed for syphilis infection at baseline and quarterly with RPR (BD Macro-Vue, USA) and TPPA (Fujirebio, Japan) testing up to 24 months. A dacron swab was used to collect exudate from chancre-like lesions and placed into $500 \mu \mathrm{L}$ of lysis buffer. DNA extraction was performed using QIAamp mini kit (Qiagen, Valencia, CA). Using specific primers for $\mathrm{Tp} 47$ region target, an aliquot of the DNA sample was amplified using conventional PCR. Subtyping of T. pallidum TP47 positives was performed using detection of number of 60 -bp tandem repeats in the arp gene and analysis by RFLP of 3 tpr genes (TprE, G, J) according to CDC guidelines.

Results Among 401 participants, 26 presented with primary syphilitic lesions at baseline or follow-up with RPR (TPPA confirmed) titers ranging from 1:2-1:64. Of those 26 total lesions, $1(7 \%)$ of 14 tested was dark-field positive. TP DNA screening using TP47 PCR yielded 12 (44.4\%) positives. Among eight typable, four were subypes $14 \mathrm{~d}(33.3 \%)$, two $15 \mathrm{~d}(16.7 \%)$, one $16 \mathrm{~d}(8.3 \%)$ and one $0 \mathrm{~d}(16.7 \%)$ where $0=$ non-typable arp.

Conclusion T. pallidum subtypes $14 \mathrm{~d}$ and $15 \mathrm{~d}$ were the most prevalent strains in lesions obtained from MSM/TW who had chancre-like lesions in Lima, Peru. Dark field testing results and typing difficulties could be due to low bacterial load and needs to be considered in sampling methods.

Disclosure of interest statement The study Picasso is funded by a NIAID grant and was implemented by Cayetano Heredia in collaboration with the University of California Los Ángeles. The molecular part of the study was implemented under the supervision of the University of Washington. No pharmaceutical grants were received during the development of this study.

\section{P09.35 ERADICATION OF SYPHILIS? - NOT THERE YET ONGOING PUBLIC HEALTH RESPONSE TO A SYPHILIS OUTBREAK IN THE NORTHERN TERRITORY, AUSTRALIA}

${ }^{1} \mathrm{~L}$ Garton*, ${ }^{1} \mathrm{M}$ Gunathilake, ${ }^{1} \mathrm{JY}$ Su, ${ }^{1} \mathrm{M}$ Russell, ${ }^{1} \mathrm{TW}$ Yip, ${ }^{2} \mathrm{~A}$ Hope, ${ }^{3} \mathrm{~N}$ Ryder, ${ }^{1} \mathrm{M}$ Thalanany, ${ }^{1} \mathrm{~V}$ Krause. ${ }^{1}$ Centre of Disease Control, Darwin, Northern Territory; ${ }^{2}$ Aboriginal Medical Services Alliance, Northern Territory; ${ }^{3}$ Newcastle Sexual Health Services, Hunter New England Local Health District, NSW

10.1136/sextrans-2015-052270.419

Introduction An extensive outbreak of infectious syphilis in the Northern Territory (NT) was identified in Central Australia and Katherine regions in 2014. The NT Centre for Disease Control (CDC) launched an outbreak response to contain it.

Methods A multi-disciplinary outbreak response team was formed to drive the response. Comprehensive testing and treatment data were regularly collected and used to monitor the outbreak and to guide the response measures. Opportunistic testing was proactively promoted in affected regions. In areas with high numbers of cases and untraceable contacts, a community-wide screen using a point-of-care test (PoCT) for syphilis was conducted for those aged 12-30 years. Local guidelines were followed in treatment and contact tracing. Updates on the outbreak response were communicated to the affected communities regularly.
Results As of 7 April 2015, 112 cases (60 females and 52 males) of infectious syphilis were detected, consisting of 74 and 38 confirmed and probable cases, respectively. Their ages ranged from 12 to 37 years (median: 17; inter-quartile range: 15-20.5). Community-wide screening was conducted in 2 remote Aboriginal communities with combined population coverage of $62.7 \%$ and a prevalence of $8.4 \%$ in the targeted age group (12-30 years). All positive PoCT results were confirmed by normal syphilis serology tests.

Conclusion Effective syphilis outbreak control requires up-todate surveillance on testing and treatment as well as an outbreak response team capable of adopting the best disease control measures in a timely fashion to the different stages and needs of the outbreak. In particular, using PoCT for community screening is proving to be an extremely useful case finding method, shortening the time to treatment and thus the period of infectiousness in communities most affected. Further control of the outbreak will require inter-jurisdictional collaboration.

Disclosure of interest statement There are no conflicts of interest

\section{P09.36 IMPROVING UPTAKE, INTERPRETATION AND QUALITY OF SYPHILIS TESTING IN THE AMERICAS THROUGH THE DEVELOPMENT OF A NEW REGIONAL GUIDANCE DOCUMENT}

${ }^{1}$ Freddy Perez* ${ }^{2}$ Adele Schwartz Benzaken, ${ }^{3}$ Kevin Karem, 'Jorge Matheu, ${ }^{3}$ Mary Kamb. 'HIV, Hepatitis, Tuberculosis and Sexually Transmitted Infectious Unit-Communicable Diseases and Health Analysis Department- Pan American Health Organization; 'Department of STI, AIDS and Viral Hepatitis, Health Surveillance Unit, Ministry of Health of Brazil; ${ }^{3}$ Centers for Disease Control and Prevention (CDC), Division of Sexually Transmitted Disease Prevention, National Center for HIVIAIDS, Viral Hepatitis, STD and TB Prevention

\subsection{6/sextrans-2015-052270.420}

Introduction In 2009, an initiative to eliminate mother-to-child transmission (MTCT) of HIV and congenital syphilis was launched in the Americas. The region sought to develop means of increasing uptake of syphilis testing for pregnant women and key populations at risk for syphilis to ensure prompt detection and treatment with minimal loss to follow-up.

Methods For a regional consultation, a PAHO/CDC team reviewed the published and grey literature on syphilis testing to identify regional standards and gaps. In April 2014, a regional meeting was held with representatives from health ministries and technical experts in maternal and child health and laboratory diagnostics. Participants reviewed and discussed the data, reported on best practices, and identified key areas to be included in a regional policy document.

Results Participants raised several concerns, such as a lack of regional or global guidance on syphilis testing strategies for specific clinical settings being a barrier impeding elimination of MTCT of HIV and syphilis. Also, limited integration of HIV and syphilis program and operations' systems was a "missed opportunity" to leverage efficiencies, personnel and funding in many countries. Furthermore, existing "best practices" promoting syphilis testing in different clinical settings had not been widely circulated. Participants recommended the development of a regional consensus document. In March 2015, this Guidance outlining syphilis testing algorithms in different clinical settings was published emphasising 5 areas for national programs: (1) comprehensive national policies on syphilis testing and treatment; (2) syphilis testing algorithms appropriate for specific populations or clinical (or outreach) settings, depending on laboratory capacity (e.g., rapid syphilis testing strategies); (3) 
ongoing quality assurance of syphilis testing; (4) procurement mechanisms supporting high-quality and affordable syphilis test kits and supplies; and (5) enhanced national reporting of syphilis cases.

Conclusion This first-ever Regional Guidance on syphilis testing will set standards on which future country assessments will be evaluated.

\section{P09.37 SYPHILIS RAPID TEST VALIDATION FOR TREPONEMAL DIAGNOSIS IN GUATEMALA, 2013-2014}

${ }^{1} \mathrm{E}$ Arana Flora, ${ }^{2} \mathrm{~L}$ Castillo-Signor, ${ }^{2} \mathrm{P}$ Marchorro, ${ }^{3} \mathrm{~V}$ Girón, ${ }^{2} \mathrm{~A}$ Lopez, ${ }^{2} \mathrm{C}$ Escobar, ${ }^{1} \mathrm{C}$ Vargas, ${ }^{1} \mathrm{R}$ Mendizabal-Burastero, 'S Morales-Miranda*. ${ }^{1} H I V$ Unit, Centers for Health Studies, Universidad Del Valle de Guatemala; ${ }^{2}$ National Health Laboratory, Ministry of Health, Guatemala; ${ }^{3}$ HIV National Program, Ministry of Health, Guatemala

\subsection{6/sextrans-2015-052270.421}

Background International guidelines for syphilis testing recommend non-treponemal tests, and confirmation by more complex treponemal test such as Treponema pallidum haemagglutination (TPHA), enzyme-linked immunosorbent assay (ELISA) or others. Similar to HIV rapid tests, rapid treponemal tests can improve point-of-care testing in laboratories with limited capacities. We present main results of a validation for treponemal diagnosis performed in Guatemala.

Methods A syphilis rapid test laboratory validation was performed in two phases during 2013-2014. First stage was performed using frozen serums from National Health Laboratory and second stage was performed using whole blood from pregnant women from peripheral health services from Guatemala City. Four treponemal rapid tests were evaluated in the first stage: Determine TP (DetTP), SD Syphilis (SDSyp), Omega Visitect (OVis), and Rapid Syphilis (RSyp). Only two tests were evaluated in the second stage: DetTP and SDSyp. For confirmation, TPHA was used for diagnosis of treponemal antibodies in serum and treponemal ELISA for plasma.

Results A total of 460 serums were evaluated in first stage and 432 whole blood samples in second stage. Compared to TPHA, sensitivity ranged from $87.8 \%$ for RSyp to $99.2 \%$ in DetTP in serum and $100 \%$ for SDSyp and DetTP in whole blood. Specificity was lower, ranging from $74.8 \%$ for DetTP to $87.7 \%$ for SDSyp in serum and $100 \%$ for SDSyp and DetTP in whole blood.

Conclusion Rapid treponemal tests are a useful diagnostic tool in syphilis. Sensibility was high enough to be used as a screening test, with good results in whole blood samples. Low prevalence of syphilis in the validation contributed to poor positive predictive values. The use of a reverse algorithm might be useful for implementation of this test in Guatemala.

Disclosure of interest statement We declare that we have no conflicts of interest.

\section{P09.38 SYPHILIS PREVALENCE AND RISK BEHAVIOUR AMONG PEOPLE LIVING WITH HIV IN MANAGUA, NICARAGUA: BSS-2009, BSS-2014 AND STI SENTINEL SURVEILLANCE (VICITS) 2014}

L Romero, I Loya-Montiel, A Solorzano, S Morales-Miranda. HIV Unit, Center for Health Studies, Universidad Del Valle de Guatemala

10.1136/sextrans-2015-052270.422
Background In Nicaragua, 9,739 HIV cases have been reported during 1987-2014. Two Central American Behavioural Surveillance Survey (BSS) among people living with HIV (PLHIV) to estimate prevalence of STI and risk behaviour have been conducted. Since 2012, the STI Sentinel Surveillance Strategy (VICITS) has offered STI diagnosis/treatment and behavioural intervention for PLHIV in public health sites. We present sociodemographic/behavioural and STI prevalence among PLHIV from BSS (2009 and 2014) and VICITS databases.

Methods For the BSS, we used a convenience sample of PLHIV seeking HIV services at Roberto Calderon Hospital (RCH) in Managua, from September-December 2009 and January-March 2014. For VICITS, PLHIV seeking services at RCH during 2014 were included. BSS information was obtained through Audio Computer-Assisted Self-Interview. VICITS data were abstracted from VICITS information system. Blood samples were collected for Syphilis testing according to national guidelines. Data were analysed using STATA v13.0.

Results Each BSS survey recruited 200 PLHIV (male 53.5\% BSS2009; 74.0\% BSS-2014). VICITS recruited 146 PLHIV $(85.7 \%$ male). Median age was 34 years old for all three data sources. One-third reported being married/living together in BSS-2009 and VICITS (44\%, BSS-2014). Alcohol use in the last month was reported by $33.2 \%$ in BSS-2009 and 50\% in BSS-2014 and VICITS. Condom use in the last sex with any partner was $60.0 \%$ in BSS-2009 and $75.5 \%$ in BSS-2014 and VICITS. Prevalence of syphilis was $11.6 \%$ (95\% CI: 7.5-16.8) in BSS-2009, $10.0 \%$ (95\% CI: $6.2-15.0$ ) in BSS-2014 and 16.7\% (95\% CI: 8.1-19.8) in VICITS.

Conclusion Although socio-demographic/behavioural data of PLHIV in BSS and VICITS were similar, syphilis prevalence was higher among PLHIV enrolled in VICITS. Our results indicate that VICITS strategy can provide valuable information about sexual behaviour of PLHIV. Our findings suggest the use of programmatic data in lieu of complex and expensive surveys to monitor trends in STI prevalence and sexual behaviour.

Disclosure of interest We declare that we have no conflicts of interest.

\section{P09.39 HIV/SYPHILIS PREVALENCE AND RISK BEHAVIOURS AMONG TRANSGENDER WOMEN AND MEN WHO HAVE SEX WITH MEN IN NICARAGUA: SENTINEL SURVEILLANCE AND STI CONTROL (VICITS), 2014}

R Gutiérrez, L Romero, I Loya-Montiel, A Solorzano, P Rosales, S Morales-Miranda*. HIV Unit of Center for Health Studies, Universidad Valle de Guatemala

\subsection{6/sextrans-2015-052270.423}

Background Nicaragua has a HIV concentrated epidemic, with 9.7\% among men who have sex with men and transgender women (MSM/TGW). Since 2011, Sentinel Surveillance and STI Control (VICITS) strategy has been implemented in public health clinics for key populations to provide STI diagnosis/treatment and behavioural change interventions. We describe risk sexual behaviours and HIV/syphilis prevalence among MSM/ TGW who attended at least one of two VICITS clinics.

Methods We included in the analysis: Men, $\geq 18$ years old (yo), who reported having sex with men in the last year, who attended one of the two selected VICITS clinics in Managua (Pedro Altamirano Hospital) or Granada (Sinforoso Bravo Hospital), from 1 January 2014 through 31 December 2014. Blood samples were collected for HIV and syphilis testing according to 